\title{
Utilization Pattern and Credibility upon Different Information Sources by the NFSM Beneficiaries and Non-Beneficiaries Farmers in Northern Hills Agro-Climatic Zone of Chhattisgarh
}

\author{
Akanksha Pandey ${ }^{*}$ and H.K. Awasthi \\ Department of Agriculture Extension, Indira Gandhi Krishi Vishwavidyalaya, Raipur-492012 \\ (C.G.), India \\ *Corresponding author
}

\begin{abstract}
A B S T R A C T
The present study was undertaken in four districts of Northern hills Agro-climatic zone of Chhattisgarh to know the Utilization pattern and credibility upon different sources information sources by the NFSM beneficiaries and non-beneficiaries farmers. The

Keywords

Utilization,

Information level,

Sources, Farmers,

Chhattisgarh

Article Info

Accepted:

18 August 2018

Available Online:

10 September 2018 beneficiary respondents had utilized and ranked I to the Cooperative society source of information and followed by Progressive farmer ranked II, RAEO's/ Agriculture officers ranked III and further information sources. In case of Non-beneficiaries respondents utilized and ranked I to the Progressive farmer followed by Cooperative society ranked II, Television ranked III and further sources. In case of beneficiaries respondents data shows that most $(52.60 \%)$ of respondents had medium level of information sources while, 25 per cent respondents had high level and 22.39 per cent had low level information respectively. Regarding Non-beneficiaries results shows that majority $(61.45 \%)$ respondents had medium level of information sources followed by 21.87 per cent had low level and only 16.66 per cent had high level of information. Regarding beneficiaries results inferred that the RAEO's/ Agriculture Officers (83.33\%) is most highly credible information source for the respondents and majority $(73.61 \%)$ of Non-beneficiaries respondents had credibility and ranked I to the Input dealers. Regarding beneficiary respondents had major credibility and ranked I to RAEO/ Agriculture officers and Non-beneficiaries had credibility and ranked I to the Input dealers.
\end{abstract}

\section{Introduction}

Information services can be defined as services which provide data, knowledge, and information that are of interest to users. The major function of information is to increase the knowledge of the user, to reduce his level of uncertainty or reduce the varieties of choices available to the users of information. The information should be effective, it must be accurate, timely and relevant. Sources of information is directly associated with the knowledge and adoption of any technology. These sources provide information to the respondents about different new and improved technology or practices related to different crops. For determine the information index of various sources of information, the concerned respondents were asked to assign their emphasis to all such sources which were 
utilized by them for getting agricultural information. The overall relative information index of each source was worked out with the help of following equation:

RCIi $=\frac{\text { OIIi }}{\text { TOIli }}$

Where,

RIIi= Relative information index for ith source

OIIi= Obtained information score for ith source $\{$ (No. of respondents ranked regular information to ith source $x$ 2) + (No. of respondents ranked occasional information to $i^{\text {th }}$ source $\left.\left.\mathrm{x} 1\right)\right\}$.

TOCI $=$ Total Obtainable Information score of ith source \{Total No. of respondents utilized the ith source of information $\mathrm{x} 2\}$.

By using above formula information index all the selected source of information were ranked first, second, third and so on in descending order of preference given by respondents to each mention source of information.

\section{Materials and Methods}

Chhattisgarh state has 27 districts i.e., Bijapur, Sukma, Dantewada, Bastar, Kondagaon, Narayanpur, Kanker, Kawardha, Rajnandgaon, Balod, Durg, Bemetara, Dhamtari, Gariyaband, Raipur, Baloda Bazar, Mahasamund, Bilaspur, Mungeli, Korba, Janjgir-Champa, Jashpur, Raigarh, Koriya, Surajpur, Surguja, Balrampur. Out of these, the study was conducted in Surguja, Surajpur, Jashpur and Balrampur district of Northern hills Agro-climatic zone of Chhattisgarh state during the year 2014-2015. Out of total blocks of these selected districts, 08 blocks were selected for the study. Total 288 (beneficiaries 192 and non-beneficiaries 96) farmers were selected randomly from 16 selected villages with purpose to collect the data. The data were collected personally with help of interview schedule developed for the collection of data. Collected data were analyzed with the help of suitable statistical methods.

\section{Results and Discussion}

\section{Sources of information}

The data regarding utilization of information sources for seeking the information about improved crop production technologies are incorporated in the Table 1 and utilization pattern of information sources is determined in three categories i.e. Regular, Occasional and Never.

The data contained in Table 1 shows that most $(51.56 \%)$ of beneficiaries respondents were regularly utilized progressive farmer followed TV, cooperative society, radio, Group meetings, kissan mitra, and Farmer's fair/Agril. Demonstration, Training, Agricultural Scientists and Demonstrations, Farmer visiting, Farmers day/Field day \& Agriculture magazines, Newspaper, RAEO's/ Agril. Officers, internets, friends, neighbors \& rural leader, input dealer, relatives and Kissan Call Center with 44.79, 43.23, 39.06, 37.50, $34.38,33.33,30.21,21.35,17.71,14.06$, 10.94, 9.90, 7.29, 4.17, 3.65, 3.13, 1.56 and 0.52 per cent respectively. While, majority (83.85\%) of Non-beneficiaries respondents occasionally utilized RAEO's/ Agril. officers followed by 31.25 per cent input dealers, 29.17 per cent Cooperative society, 16.15 per cent Kissan Mitra, 4.17 per cent Agricultural scientist, 3.13 per cent demonstrations \& Group meetings, very few per cent from TV, Progressive farmer, Friends, Radio, Farmer's fair/Agril. Demonstration, Training, Farmers 
day/Field day, Internet and others were not occasionally utilized by the respondents.

In case of Non-beneficiaries most (62.50\%) of respondents regularly utilized progressive farmer followed by cooperative society, television, Radio, Group meetings, Agriculture magazines, RAEO/ Agril. officers, Farmer's fair/Agril. \& Demonstration, Kissan mitra, Internet \& Training, Input dealers, Farmers day/Field day, Neighbors \& Friends, Demonstrations \& Rural leaders 53.13, 40.63, 38.54, 32.29, $25.00,22.92,16.67,14.58,13.54,11.46,7.29$, $6.25,4.17$ per cent. Only few $(2.08 \%)$ respondents regularly utilized Newspaper, Farmer visiting, Agricultural Scientists, Relatives and no respondents were utilized Kissan Call Center. Whereas, most (54.17\%) of the respondents were occasionally utilized Input dealers followed by 18.75 per cent Cooperative society, 16.67 per cent RAEO/ Agril. Officers, 9.38 per cent Kissan mitra, 6.25 per cent Progressive farmer very few per cent from Television, Farmer's fair/Agril., Demonstration, Training, and others were not occasionally utilized by the respondents.

The present findings are supported by the findings of Sekhar C.S.C. and Bhatt (2012) revealed that the main sources of knowledge or information about the NFSM in the district are extension agent. Niranjan et al., (2016) found that the major source of awareness of NFSM among the sample beneficiaries was found to be Agriculture Department (99.7\%). Also the findings of Singh V. 2017 reported that in the study area, RAEO ranked first being utilized by 86.67 per cent of respondents. The study also reveals that 66.67 per cent of the respondents had obtained the information from progressive farmers, followed by 45.83 per cent of respondents obtained the information from telephones, neighbours/ friends (41.67\%), T.V. (37.50\%), (KCC) Kissan call centers (25.00\%), SADO
(20.83\%), training $(18.33 \%)$ were other popular sources of information.

\section{Source of information index}

Regarding further analysis source of information index was worked out. A close observation of data is presented in Table 2 indicates that majority $(57.81 \%)$ of beneficiary respondents utilized and ranked I to the Cooperative society source of information and followed by Progressive farmer (52.34\%, ranked II), RAEO's/ Agril. officers (51.82\%, ranked III), Television (46.09\% ranked IV), Kissan mitra $(42.45 \%$ ranked V), Radio 39.84\% ranked VI), Group meetings (39.06\% ranked VII), Farmer's fair/Agril. Demonstration $33.85 \%$ ranked VIII), Training (30.99 \% ranked IX), Agricultural Scientists (23.44\% ranked X), Demonstrations (22.92\% ranked XI), Input dealers (18.75\% ranked XII), Farm visiting ( $17.71 \%$ ranked XIII), Farmers day/Field day (14.58\% ranked XIV), Agriculture magazines (14.32\% ranked XV), Newspaper $(10.94 \%$ ranked XVI), Internet (7.81\% ranked XVII), Friends $(4.43 \%$ ranked XVIII), Neighbors, Rural leaders (3.65\% ranked XIX) and Kissan Call Center (0.52 \% ranked XX) respectively.

In case of Non-beneficiaries respondents, result shows that majority $(65.63 \%)$ of respondents utilized and ranked $\mathrm{I}$ to the Progressive farmer followed by Cooperative society (62.50\% ranked II), Television (41.15 $\%$ ranked III), Radio, Input dealers (38.54\% ranked IV), Group meetings (32.29\% ranked V), RAEO's/ Agril. officers (31.25\% ranked VI), Agriculture magazines $(25.00 \%$ ranked VII), Kissan mitra (19.27\% ranked VIII), Farmer's fair/Agril. Demonstration (17.19\% ranked IX), Training (14.06\% ranked X), Internet (13.54 \% ranked XI), Farmers day/Field day (7.29 \% ranked XII), Friends, Neighbors $(6.25 \%$ ranked XIII), 
Demonstrations, Rural leaders (4.17\% ranked XIV), Farm visiting, Newspaper, Agricultural Scientists, Relatives (2.08\% ranked XV) respectively and Kissan Call Center was not utilized by the respondents.

In the light of above view it can be concluded that beneficiary respondents utilized and ranked I to the Cooperative society and nonbeneficiary's respondent ranked I to the Progressive farmer.

\section{Overall level of information sources}

To get an overview of the overall level of information sources, the respondents were classified into three categories i.e. Low, Medium and High on the basis of calculated mean and standard deviation of the obtained scores by the respondents. The distribution of respondents in each category is given in Table 4.

In case of beneficiaries respondents data shows that most $(52.60 \%)$ of respondents had medium level of information sources while, 25 per cent respondents had high level and 22.39 per cent had low level information respectively. Regarding Non-beneficiaries results shows that majority $(61.45 \%)$ respondents had medium level of information sources followed by 21.87 per cent had low level and only 16.66 per cent had high level of information. From the above results, it can be concluded that majority of the beneficiary and non-beneficiary had medium level of information but most of the beneficiary respondents also had high level of information while Non-beneficiaries were had very minimum per cent of high level source of information.

\section{Credibility of information sources}

The credibility refers to the perceived trustworthiness and expertise accorded to a source or channel by its audience at any given time. Therefore, sources and channels of agriculture information play major role in diffusion of agriculture innovation.

The credibility of information sources and channels affects the extent of adoption of different improved agricultural practices by the farmers. The credibility of information sources were measured in three categories i.e. highly credible, moderately credible and least credible and results are presented in Table 3.

Regarding beneficiaries, results inferred that the RAEO's/Agril. Officers (83.33\%) is most highly credible information source for the respondents followed by 31.77 per cent Input dealers, 30.73 per cent Cooperative society, 20.83 per cent Kissan mitra, 14.06 per cent Agricultural Scientists, 8.85 per cent Training, 6.25 per cent Group meetings, 4.69 per cent Demonstrations, 3.65 per cent Progressive farmer, few per cent credibility in Farmer's fair/Agril. Demonstration, Television, Radio, Agriculture magazines and Farmer visiting. Whereas, others information sources are not highly credible for respondents.

About 49.48 per cent Progressive farmer are moderately credible for beneficiaries respondents followed by Television $(46.88 \%)$, Radio (40.10\%), Group meetings (34.38\%), Farmer's fair/Agril. Demonstration (32.29\%), Kissan mitra (29.69\%), Training (22.92\%), Demonstrations (19.79\%), Farmer visiting (16.67\%), Cooperative society (15.63\%), Farmers day/Field day (15.10\%), Agriculture magazines (14.06\%), Agricultural Scientists (11.46\%), Newspaper \& RAEO's / Agril. officers $(10.42 \%)$, Internet $(8.33 \%)$, Friends (4.17\%), Neighbors, Rural leaders $(3.65 \%)$ and Input dealers (2.60\%) respectively. Relatives and Kissan Call Center sources are very few per cent of moderately credible for respondents. 
Table.1 Distribution of the respondents according to their utilization pattern of information from different sources

\begin{tabular}{|c|c|c|c|c|c|c|c|c|c|c|c|c|c|c|c|c|c|c|c|}
\hline \multirow{4}{*}{$\begin{array}{l}\text { SI. } \\
\text { No }\end{array}$} & \multirow{4}{*}{ Information sources } & \multicolumn{18}{|c|}{ Utilization pattern of different sources of information } \\
\hline & & \multicolumn{6}{|c|}{ Beneficiaries $n=192$} & \multicolumn{6}{|c|}{ Non-beneficiaries $n=96$} & \multicolumn{6}{|c|}{ Overall respondents $n=288$} \\
\hline & & \multicolumn{2}{|c|}{ Regular } & \multicolumn{2}{|c|}{ Occasional } & \multicolumn{2}{|c|}{ Never } & \multicolumn{2}{|c|}{ Regular } & \multicolumn{2}{|c|}{ Occasional } & \multicolumn{2}{|c|}{ Never } & \multicolumn{2}{|c|}{ Regular } & \multicolumn{2}{|c|}{ Occasional } & \multicolumn{2}{|c|}{ Never } \\
\hline & & $\mathrm{F}$ & $\mathrm{P}$ & $\mathrm{F}$ & $\mathrm{P}$ & $\mathrm{F}$ & $\mathrm{P}$ & $\mathrm{F}$ & $\mathrm{P}$ & $\mathrm{F}$ & $\mathrm{P}$ & $\mathrm{F}$ & $\mathrm{P}$ & $\mathrm{F}$ & $\mathrm{P}$ & $\mathrm{F}$ & $\mathrm{P}$ & $\mathrm{F}$ & $\mathrm{P}$ \\
\hline 1 & Progressive farmer & 99 & 51.56 & 3 & 1.56 & 90 & 46.88 & 60 & 62.50 & 6 & 6.25 & 30 & 31.25 & 159 & 55.21 & 9 & 3.13 & 120 & 41.67 \\
\hline 2 & Neighbors & 7 & 3.65 & 0 & 0.00 & 185 & 96.35 & 6 & 6.25 & 0 & 0.00 & 90 & 93.75 & 13 & 4.51 & 0 & 0.00 & 275 & 95.49 \\
\hline 3 & Friends & 8 & 4.17 & 1 & 0.52 & 183 & 95.31 & 6 & 6.25 & 0 & 0.00 & 90 & 93.75 & 14 & 4.86 & 1 & 0.35 & 273 & 94.79 \\
\hline 4 & Relatives & 3 & 1.56 & 0 & 0.00 & 189 & 98.44 & 2 & 2.08 & 0 & 0.00 & 94 & 97.92 & 5 & 1.74 & 0 & 0.00 & 283 & 98.26 \\
\hline 5 & Rural leaders & 7 & 3.65 & 0 & 0.00 & 185 & 96.35 & 4 & 4.17 & 0 & 0.00 & 92 & 95.83 & 11 & 3.82 & 0 & 0.00 & 277 & 96.18 \\
\hline 6 & Cooperative society & 83 & 43.23 & 56 & 29.17 & 53 & 27.60 & 51 & 53.13 & 18 & 18.75 & 27 & 28.13 & 134 & 46.53 & 74 & 25.69 & 80 & 27.78 \\
\hline 7 & Agriculture magazines & 27 & 14.06 & 1 & 0.52 & 164 & 85.42 & 24 & 25.00 & 0 & 0.00 & 72 & 75.00 & 51 & 17.71 & 1 & 0.35 & 236 & 81.94 \\
\hline 8 & Radio & 75 & 39.06 & 3 & 1.56 & 114 & 59.38 & 37 & 38.54 & 0 & 0.00 & 59 & 61.46 & 112 & 38.89 & 3 & 1.04 & 173 & 60.07 \\
\hline 9 & Television & 86 & 44.79 & 5 & 2.60 & 101 & 52.60 & 39 & 40.63 & 1 & 1.04 & 56 & 58.33 & 125 & 43.40 & 6 & 2.08 & 157 & 54.51 \\
\hline 10 & $\begin{array}{l}\text { Farmer's fair/Agril. } \\
\text { Demonstration }\end{array}$ & 64 & 33.33 & 2 & 1.04 & 126 & 65.63 & 16 & 16.67 & 1 & 1.04 & 79 & 82.29 & 80 & 27.78 & 3 & 1.04 & 205 & 71.18 \\
\hline 11 & Training & 58 & 30.21 & 3 & 1.56 & 131 & 68.23 & 13 & 13.54 & 1 & 1.04 & 82 & 85.42 & 71 & 24.65 & 4 & 1.39 & 213 & 73.96 \\
\hline 12 & RAEO/ Agril. Officers & 19 & 9.90 & 161 & 83.85 & 12 & 6.25 & 22 & 22.92 & 16 & 16.67 & 58 & 60.42 & 41 & 14.24 & 177 & 61.46 & 70 & 24.31 \\
\hline 13 & Agricultural Scientists & 41 & 21.35 & 8 & 4.17 & 143 & 74.48 & 2 & 2.08 & 0 & 0.00 & 94 & 97.92 & 43 & 14.93 & 8 & 2.78 & 237 & 82.29 \\
\hline 14 & Farmers day/Field day & 27 & 14.06 & 2 & 1.04 & 163 & 84.90 & 7 & 7.29 & 0 & 0.00 & 89 & 92.71 & 34 & 11.81 & 2 & 0.69 & 252 & 87.50 \\
\hline 15 & Farmer visiting & 34 & 17.71 & 0 & 0.00 & 158 & 82.29 & 2 & 2.08 & 0 & 0.00 & 94 & 97.92 & 36 & 12.50 & 0 & 0.00 & 252 & 87.50 \\
\hline 16 & Demonstrations & 41 & 21.35 & 6 & 3.13 & 145 & 75.52 & 4 & 4.17 & 0 & 0.00 & 92 & 95.83 & 45 & 15.63 & 6 & 2.08 & 237 & 82.29 \\
\hline 17 & Kissan mitra & 66 & 34.38 & 31 & 16.15 & 95 & 49.48 & 14 & 14.58 & 9 & 9.38 & 73 & 76.04 & 80 & 27.78 & 40 & 13.89 & 168 & 58.33 \\
\hline 18 & News paper & 21 & 10.94 & 0 & 0.00 & 171 & 89.06 & 2 & 2.08 & 0 & 0.00 & 94 & 97.92 & 23 & 7.99 & 0 & 0.00 & 265 & 92.01 \\
\hline 19 & Kissan Call Center & 1 & 0.52 & 0 & 0.00 & 191 & 99.48 & 0 & 0.00 & 0 & 0.00 & 96 & 100 & 1 & 0.35 & 0 & 0.00 & 287 & 99.65 \\
\hline 20 & Internet & 14 & 7.29 & 2 & 1.04 & 176 & 91.67 & 13 & 13.54 & 0 & 0.00 & 83 & 86.46 & 27 & 9.38 & 2 & 0.69 & 259 & 89.93 \\
\hline 21 & Group meetings & 72 & 37.50 & 6 & 3.13 & 114 & 59.38 & 31 & 32.29 & 0 & 0.00 & 65 & 67.71 & 103 & 35.76 & 6 & 2.08 & 179 & 62.15 \\
\hline 22 & Input dealers & 6 & 3.13 & 60 & 31.25 & 126 & 65.63 & 11 & 11.46 & 52 & 54.17 & 33 & 34.38 & 17 & 5.90 & 112 & 38.89 & 159 & 55.21 \\
\hline
\end{tabular}

*Multiple responses, $\mathrm{F}=$ Frequency and $\mathrm{P}=$ Percentage 
Table.2 Utilization index of various information sources by the respondents

\begin{tabular}{|r|l|c|c|c|c|c|c|}
\hline $\begin{array}{l}\text { SI. } \\
\text { No. }\end{array}$ & Information sources & \multicolumn{3}{|c|}{$\begin{array}{c}\text { Beneficiaries } \\
\mathbf{n = 1 9 2}\end{array}$} & \multicolumn{2}{|c|}{$\begin{array}{c}\text { Non-beneficiaries } \\
\mathbf{n = 9 6}\end{array}$} & \multicolumn{2}{|c|}{$\begin{array}{c}\text { Overall respondents } \\
\text { n=288 }\end{array}$} \\
\hline & & Index & Rank & Index & Rank & Index & Rank \\
\hline $\mathbf{1}$ & Progressive farmer & 52.34 & II & 65.63 & I & 56.77 & II \\
\hline $\mathbf{2}$ & Neighbors & 3.65 & XIX & 6.25 & XIII & 4.51 & XVIII \\
\hline $\mathbf{3}$ & Friends & 4.43 & XVIII & 6.25 & XIII & 5.03 & XVII \\
\hline $\mathbf{4}$ & Relatives & 1.56 & XX & 2.08 & XV & 1.74 & XX \\
\hline $\mathbf{5}$ & Rural leaders & 3.65 & XIX & 4.17 & XIV & 3.82 & XIX \\
\hline $\mathbf{6}$ & Cooperative society & 57.81 & I & 62.50 & II & 59.38 & I \\
\hline $\mathbf{7}$ & Agriculture magazines & 14.32 & XV & 25.00 & VII & 17.88 & X \\
\hline $\mathbf{8}$ & Radio & 39.84 & VI & 38.54 & IV & 39.41 & V \\
\hline $\mathbf{9}$ & Television & 46.09 & IV & 41.15 & III & 44.44 & IV \\
\hline $\mathbf{1 0}$ & Farmer's fair/Agril. & 33.85 & VIII & 17.19 & IX & 28.30 & VIII \\
\hline & Demonstration & & & & & & \\
\hline $\mathbf{1 1}$ & Training & 30.99 & IX & 14.06 & X & 25.35 & IX \\
\hline $\mathbf{1 2}$ & RAEO's/ Agril. officers & 51.82 & III & 31.25 & VI & 44.97 & III \\
\hline $\mathbf{1 3}$ & Agricultural Scientists & 23.44 & X & 2.08 & XV & 16.32 & XII \\
\hline $\mathbf{1 4}$ & Farmers day/Field day & 14.58 & XIV & 7.29 & XII & 12.15 & XIV \\
\hline $\mathbf{1 5}$ & Farm visiting & 17.71 & XIII & 2.08 & XV & 12.50 & XIII \\
\hline $\mathbf{1 6}$ & Demonstrations & 22.92 & XI & 4.17 & XIV & 16.67 & XI \\
\hline $\mathbf{1 7}$ & Kissan mitra & 42.45 & V & 19.27 & VIII & 34.72 & VII \\
\hline $\mathbf{1 8}$ & News paper & 10.94 & XVI & 2.08 & XV & 7.99 & XVI \\
\hline $\mathbf{1 9}$ & Kissan Call Center & 0.52 & XX & 0.00 & 0 & 0.35 & XXI \\
\hline $\mathbf{2 0}$ & Internet & 7.81 & XVII & 13.54 & XI & 9.72 & XV \\
\hline $\mathbf{2 1}$ & Group meetings & 39.06 & VII & 32.29 & V & 36.81 & VI \\
\hline $\mathbf{2 2}$ & Input dealers & 18.75 & XII & 38.54 & IV & 25.35 & IX \\
\hline & & & & & & \\
\hline
\end{tabular}


Table.3 Distribution of the respondents according to the credibility of information sources

\begin{tabular}{|c|c|c|c|c|c|c|c|c|c|c|c|c|c|c|c|c|c|c|c|}
\hline \multirow{4}{*}{$\begin{array}{l}\text { SI. } \\
\text { No }\end{array}$} & \multirow{4}{*}{ Information sources } & \multicolumn{18}{|c|}{ Credibility of different sources of information } \\
\hline & & \multicolumn{6}{|c|}{ Beneficiaries $n=192$} & \multicolumn{6}{|c|}{ Non-beneficiaries $\mathrm{n}=96$} & \multicolumn{6}{|c|}{ Overall respondents $n=288$} \\
\hline & & \multicolumn{2}{|c|}{ High } & \multicolumn{2}{|c|}{ Moderate } & \multicolumn{2}{|c|}{ least } & \multicolumn{2}{|c|}{ High } & \multicolumn{2}{|c|}{ Moderate } & \multicolumn{2}{|c|}{ least } & \multicolumn{2}{|c|}{ High } & \multicolumn{2}{|c|}{ Moderate } & \multicolumn{2}{|c|}{ least } \\
\hline & & $\mathrm{F}$ & $\mathrm{P}$ & $\mathrm{F}$ & $\mathrm{P}$ & $\mathrm{F}$ & $\mathrm{P}$ & $\mathrm{F}$ & $\mathrm{P}$ & $\mathrm{F}$ & $\mathrm{P}$ & $\mathrm{F}$ & $\mathrm{P}$ & $\mathrm{F}$ & $\mathrm{P}$ & $\mathrm{F}$ & $\mathrm{P}$ & $\mathrm{F}$ & $\mathrm{P}$ \\
\hline 1 & Progressive farmer & 7 & 3.65 & 95 & 49.48 & 90 & 46.88 & 10 & 10.42 & 56 & 58.33 & 30 & 31.25 & 17 & 5.90 & 151 & 52.43 & 120 & 41.67 \\
\hline 2 & Neighbors & 0 & 0.00 & 7 & 3.65 & 185 & 96.35 & 0 & 0.00 & 6 & 6.25 & 90 & 93.75 & 0 & 0.00 & 13 & 4.51 & 275 & 95.49 \\
\hline 3 & Friends & 0 & 0.00 & 8 & 4.17 & 184 & 95.83 & 0 & 0.00 & 6 & 6.25 & 90 & 93.75 & 0 & 0.00 & 15 & 5.21 & 273 & 94.79 \\
\hline 4 & Relatives & 0 & 0.00 & 3 & 1.56 & 189 & 98.44 & 0 & 0.00 & 2 & 2.08 & 94 & 97.92 & 0 & 0.00 & 5 & 1.74 & 283 & 98.26 \\
\hline 5 & Rural leaders & 0 & 0.00 & 7 & 3.65 & 185 & 96.35 & 0 & 0.00 & 4 & 4.17 & 92 & 95.83 & 0 & 0.00 & 11 & 3.82 & 277 & 96.18 \\
\hline 6 & Cooperative society & 59 & 30.73 & 30 & 15.63 & 103 & 53.65 & 22 & 22.92 & 48 & 50.00 & 26 & 27.08 & 80 & 27.78 & 128 & 44.44 & 80 & 27.78 \\
\hline 7 & Agricultural magazines & 1 & 0.52 & 27 & 14.06 & 164 & 85.42 & 0 & 0.00 & 24 & 25.00 & 72 & 75.00 & 1 & 0.35 & 51 & 17.71 & 236 & 81.94 \\
\hline 8 & Radio & 1 & 0.52 & 77 & 40.10 & 114 & 59.38 & 0 & 0.00 & 37 & 38.54 & 59 & 61.46 & 1 & 0.35 & 114 & 39.58 & 173 & 60.07 \\
\hline 9 & Television & 1 & 0.52 & 90 & 46.88 & 101 & 52.60 & 1 & 1.04 & 38 & 39.58 & 57 & 59.38 & 2 & 0.69 & 128 & 44.44 & 158 & 54.86 \\
\hline 10 & $\begin{array}{l}\text { Farmer's fair/Agril. } \\
\text { Demonstration }\end{array}$ & 3 & 1.56 & 62 & 32.29 & 127 & 66.15 & 3 & 3.13 & 14 & 14.58 & 79 & 82.29 & 6 & 2.08 & 76 & 26.39 & 206 & 71.53 \\
\hline 11 & Training & 17 & 8.85 & 44 & 22.92 & 131 & 68.23 & 1 & 1.04 & 13 & 13.54 & 82 & 85.42 & 18 & 6.25 & 57 & 19.79 & 213 & 73.96 \\
\hline 12 & RAEO's / Agril. Officers & 160 & 83.33 & 20 & 10.42 & 12 & 6.25 & 19 & 19.79 & 19 & 19.79 & 58 & 60.42 & 179 & 62.15 & 39 & 13.54 & 70 & 24.31 \\
\hline 13 & Agricultural Scientists & 27 & 14.06 & 22 & 11.46 & 143 & 74.48 & 1 & 1.04 & 1 & 1.04 & 94 & 97.92 & 28 & 9.72 & 23 & 7.99 & 237 & 82.29 \\
\hline 14 & Farmers day/Field day & 0 & 0.00 & 29 & 15.10 & 163 & 84.90 & 1 & 1.04 & 6 & 6.25 & 89 & 92.71 & 1 & 0.35 & 35 & 12.15 & 252 & 87.50 \\
\hline 15 & Farmer visiting & 1 & 0.52 & 32 & 16.67 & 159 & 82.81 & 1 & 1.04 & 1 & 1.04 & 94 & 97.92 & 2 & 0.69 & 34 & 11.81 & 252 & 87.50 \\
\hline 16 & Demonstrations & 9 & 4.69 & 38 & 19.79 & 145 & 75.52 & 1 & 1.04 & 3 & 3.13 & 92 & 95.83 & 10 & 3.47 & 41 & 14.24 & 237 & 82.29 \\
\hline 17 & Kissan mitra & 40 & 20.83 & 57 & 29.69 & 95 & 49.48 & 9 & 9.38 & 14 & 14.58 & 73 & 76.04 & 49 & 17.01 & 71 & 24.65 & 168 & 58.33 \\
\hline 18 & News paper & 0 & 0.00 & 20 & 10.42 & 172 & 89.58 & 0 & 0.00 & 2 & 2.08 & 94 & 97.92 & 0 & 0.00 & 23 & 7.99 & 265 & 92.01 \\
\hline 19 & Kissan Call Center & 0 & 0.00 & 1 & 0.52 & 191 & 99.48 & 0 & 0.00 & 0 & 0.00 & 96 & 100 & 0 & 0.00 & 1 & 0.35 & 287 & 99.65 \\
\hline 20 & Internet & 0 & 0.00 & 16 & 8.33 & 176 & 91.67 & 0 & 0.00 & 13 & 13.54 & 83 & 86.46 & 0 & 0.00 & 29 & 10.07 & 259 & 89.93 \\
\hline 21 & Group meetings & 12 & 6.25 & 66 & 34.38 & 114 & 59.38 & 2 & 2.08 & 29 & 30.21 & 65 & 67.71 & 14 & 4.86 & 96 & 33.33 & 178 & 61.81 \\
\hline 22 & Input dealers & 61 & 31.77 & 5 & 2.60 & 126 & 65.63 & 53 & 55.21 & 10 & 10.42 & 33 & 34.38 & 114 & 39.58 & 15 & 5.21 & 159 & 55.21 \\
\hline
\end{tabular}

*Multiple responses, $\mathrm{F}=$ Frequency and $\mathrm{P}=$ Percentage 
Table.4 Distribution of the respondents according to their overall utilization level of information sources

\begin{tabular}{|c|c|c|c|c|c|c|c|}
\hline $\begin{array}{l}\text { SI. } \\
\text { No. }\end{array}$ & $\begin{array}{c}\text { Overall level of } \\
\text { information }\end{array}$ & \multicolumn{2}{|c|}{$\begin{array}{c}\text { Beneficiaries } \\
\mathbf{n = 1 9 2}\end{array}$} & \multicolumn{2}{|c|}{$\begin{array}{c}\text { Non-beneficiaries } \\
\mathbf{n = 9 6}\end{array}$} & \multicolumn{2}{c|}{$\begin{array}{c}\text { Overall respondents } \\
\mathbf{n = 2 8 8}\end{array}$} \\
\hline & & $\mathrm{F}$ & $\mathrm{P}$ & $\mathrm{F}$ & $\mathrm{P}$ & $\mathrm{F}$ & $\mathrm{P}$ \\
\hline $\mathbf{1}$ & Low & 43 & 22.39 & 21 & 21.87 & 64 & 22.22 \\
\hline $\mathbf{2}$ & Medium & 101 & 52.60 & 59 & 61.45 & 160 & 55.55 \\
\hline $\mathbf{3}$ & High & 48 & 25.00 & 16 & 16.66 & 64 & 22.22 \\
\hline
\end{tabular}

Table.5 Credibility index of various information sources by the respondents.

\begin{tabular}{|c|l|c|c|c|c|c|c|}
\hline SI. No & Information sources & \multicolumn{2}{|c|}{$\begin{array}{c}\text { Beneficiaries } \\
\mathbf{n = 1 9 2}\end{array}$} & \multicolumn{2}{|c|}{$\begin{array}{c}\text { Non-beneficiaries } \\
\text { n=96 }\end{array}$} & \multicolumn{2}{c|}{$\begin{array}{c}\text { Overall } \\
\text { respondents n=288 }\end{array}$} \\
\hline & & Index & Rank & Index & Rank & Index & Rank \\
\hline $\mathbf{1}$ & Progressive farmer & 52.26 & V & 59.72 & III & 54.75 & IV \\
\hline $\mathbf{2}$ & Neighbors & 34.55 & XVIII & 35.42 & XIV & 34.84 & XIX \\
\hline $\mathbf{3}$ & Friends & 34.72 & XVII & 35.42 & XIV & 35.07 & XVIII \\
\hline $\mathbf{4}$ & Relatives & 33.85 & XIX & 34.03 & XVIII & 33.91 & XXI \\
\hline $\mathbf{5}$ & Rural leaders & 34.55 & XVIII & 34.72 & XVI & 34.61 & XXI \\
\hline $\mathbf{6}$ & Cooperative society & 59.03 & II & 65.28 & II & 66.67 & II \\
\hline $\mathbf{7}$ & Agriculture magazines & 38.37 & XIV & 41.67 & IX & 39.47 & XIII \\
\hline $\mathbf{8}$ & Radio & 47.05 & VIII & 46.18 & VI & 46.76 & VIII \\
\hline $\mathbf{9}$ & Television & 49.31 & VI & 47.22 & V & 48.61 & VI \\
\hline $\mathbf{1 0}$ & Farmer's fair/Agril. & 45.14 & XI & 40.28 & X & 43.52 & X \\
\hline & Demonstration & & & & & & \\
\hline $\mathbf{1 1}$ & Training & 46.88 & IX & 38.54 & XI & 44.10 & IX \\
\hline $\mathbf{1 2}$ & RAEO/ Agril. officers & 92.36 & I & 53.13 & IV & 79.28 & I \\
\hline $\mathbf{1 3}$ & Agricultural Scientists & 46.53 & X & 34.38 & XVII & 42.48 & XI \\
\hline $\mathbf{1 4}$ & Farmers day/Field day & 38.37 & XIV & 36.11 & XIII & 37.62 & XV \\
\hline $\mathbf{1 5}$ & Farmer visiting & 39.24 & XIII & 34.38 & XVII & 37.73 & XIV \\
\hline $\mathbf{1 6}$ & Demonstrations & 43.06 & XII & 35.07 & XV & 40.39 & XII \\
\hline $\mathbf{1 7}$ & Kissan mitra & 57.12 & III & 44.44 & VIII & 52.89 & V \\
\hline $\mathbf{1 8}$ & News paper & 36.81 & XV & 34.03 & XVIII & 36.00 & XVII \\
\hline $\mathbf{1 9}$ & Kissan Call Center & 33.51 & XX & 0.00 & XIX & 33.45 & XXII \\
\hline $\mathbf{2 0}$ & Internet & 36.11 & XVI & 37.85 & XII & 36.69 & XVI \\
\hline $\mathbf{2 1}$ & Group meetings & 48.96 & VII & 44.79 & VII & 47.69 & VII \\
\hline $\mathbf{2 2}$ & Input dealers & 55.38 & IV & 73.61 & I & 61.46 & III \\
\hline
\end{tabular}


Table.6 Distribution of respondents according to overall credibility of information sources

\begin{tabular}{|c|c|c|c|c|c|c|c|}
\hline SI. No. & $\begin{array}{c}\text { Overall level of } \\
\text { information }\end{array}$ & \multicolumn{2}{|c|}{$\begin{array}{c}\text { Beneficiaries } \\
\mathbf{n = 1 9 2}\end{array}$} & \multicolumn{2}{|c|}{$\begin{array}{c}\text { Non-beneficiaries } \\
\mathbf{n = 9 6}\end{array}$} & \multicolumn{2}{|c|}{$\begin{array}{c}\text { Overall } \\
\text { respondents n=288 }\end{array}$} \\
\hline & & $\mathrm{F}$ & $\mathrm{P}$ & $\mathrm{F}$ & $\mathrm{P}$ & $\mathrm{F}$ & $\mathrm{P}$ \\
\hline $\mathbf{1}$ & Low & 49 & 25.52 & 2 & 2.08 & 51 & 17.70 \\
\hline $\mathbf{2}$ & Medium & 87 & 45.31 & 75 & 78.12 & 162 & 56.25 \\
\hline $\mathbf{3}$ & High & 56 & 29.16 & 19 & 19.79 & 75 & 26.04 \\
\hline
\end{tabular}

In case of Non-beneficiaries, Input dealers are most $(55.21 \%)$ highly credible information sources followed by Cooperative society (22.92\%), RAEO's / Agril. officers (19.79\%), Progressive farmer $(10.42 \%)$, Kissan mitra $(9.38 \%)$, Farmer's fair/Agril.

Demonstration (3.13\%), Group meetings $(2.08 \%)$, and some were very few $(1.04 \%)$ highly credible i.e. Television, Training, Agricultural Scientists, Farmers day/Field day, Farmer visiting, Demonstrations. Other information sources were not highly credible for Non-beneficiaries respondents.

Regarding moderately credible information resources Progressive farmer are most $(58.33 \%)$ credible followed by Cooperative society $(50 \%)$, Television $(39.58 \%)$, Radio (38.54\%), Group meeting (30.21\%), Agricultural magazines (25\%), RAEO's / Agril. officers (19.79\%), Farmer's fair/Agril. Demonstration \& Kissan mitra (14.58\%), Internet \& Training (13.54\%), Input dealers (10.42\%), Farmers day/Field day, Friends, Neighbors $(6.25 \%)$, Rural leaders $(4.17 \%)$, Demonstrations (3.13\%), Newspaper, Relatives (2.08\%), Agricultural Scientists, Farmer visiting (1.04\%) and Kissan Call Center not moderately credible for Nonbeneficiaries respondents.

The above findings are supported by the findings of Painkra V. (2014) reported that that agriculture scientists and training were fully credible source of information amongst the respondents. Senior Agriculture
Development Officer (SADOs) and Rural Agriculture Extension Officer (RAEOs) were having more than 95 per cent credibility among the respondents.

Progressive farmers, Television and Radio were also well credible sources among the respondents with 93.48, 91.67 and 90 per cent credibility, respectively.

\section{Credibility index of information sources}

For further analysis, credibility index of information sources is worked out on the basis of index per cent source of information was raked and presented in Table 5.

The data recorded in Table 5. Shows that majority 92.36 per cent beneficiary respondents had credibility and ranked I to RAEO/ Agriculture officers followed by Cooperative society $(59.03 \%$ ranked II), Kissan mitra $(57.12 \%$ ranked III), Input dealers (55.38\% ranked IV), Progressive farmer $(52.26 \%$ ranked $\mathrm{V})$, Television $(49.31 \%$ ranked VI), Group meetings (48.96\% ranked VII), Radio (47.05\% ranked VIII), Training (46.88\% ranked IX), Agricultural Scientists (46.53\% ranked X), Farmer's fair/Agril. Demonstration (45.14\% ranked XI), Demonstrations $(43.06 \%$ ranked XII), Farmer visiting (39.24\% ranked XIII), Farmers day/Field day, Agriculture magazines (38.37\% ranked XIV), Newspaper $(36.81 \%$ ranked $\mathrm{XV})$, Internet (36.11\% ranked XVI), Friends (34.72\% ranked XVII), Rural leaders, Neighbors (34.55\% ranked XVIII), Relatives 
(33.85\% ranked XIX) and Kissan Call Center $(33.51 \%$ ranked $\mathrm{XX})$ respectively.

In case of Non-beneficiaries majority $(73.61 \%)$ of respondents had credibility and ranked I to the Input dealers followed by Cooperative society $(65.28 \%$ ranked II), Progressive farmer $(59.72 \%$ ranked III), RAEO/ Agril. officers (53.13\% ranked IV), Television (47.22\% ranked V), Radio (46.18\% ranked VI), Group meetings (44.79\% ranked VII), Kissan mitra $(44.44 \%$ ranked VIII), Agriculture magazines (41.67\% ranked IX), Farmer's fair/Agril. Demonstration (40.28\% ranked X), Training (38.54\% ranked XI), Internet (37.85\% ranked XII), Farmers day/Field day (36.11\% ranked XIII), Friends, Neighbors (35.42\% ranked XIV), Demonstrations (35.07\% ranked XV), Rural leaders $(34.72 \%$ ranked XVI), Agricultural Scientists, Farmer visiting (34.38\% ranked XVII), Newspaper, Relatives (34.03\% ranked XVIII) respectively and the lastly Kissan Call Center is ranked XIX.

In the light of above view it can be concluded that beneficiary respondents had major credibility and ranked I to RAEO/ Agril. officers and Non-beneficiaries had credibility and ranked I to the Input dealers. It is may be because in study area extension linkage between agricultural officers and farmer was not very good.

\section{Overall Credibility of information sources}

To get an overview of the overall credibility of information sources, the respondents were classified on the basis of calculated mean and standard deviation into three categories, i.e. Low, Medium and High and obtained scores by the respondents. The distribution of respondents in each category is given in Table 6.

In case of beneficiaries respondents data refers that most $(45.31 \%)$ of respondents had medium credibility on information sources whereas, 29.16 per cent respondents had high credibility level and 25.52 per cent had low credibility on information sources respectively. Regarding Non-beneficiaries results shows that majority (78.12\%) respondents had medium credibility level on information sources followed by 19.79 per cent had high level credibility and only 2.08 per cent had low level credibility on information sources.

In the light of the above findings, it may be concluded that, there is many differences shown in utilization of information from different sources by the farmers. The beneficiary respondents had utilized and ranked I to the Cooperative society source of information and followed by Progressive farmer ranked II, RAEO's/ Agril. officers ranked III and further information sources. In case of Non-beneficiaries respondents utilized and ranked $\mathrm{I}$ to the Progressive farmer followed by Cooperative society ranked II, Television ranked III and further sources. Beneficiary respondents had major credibility and ranked I to RAEO/ Agril. officers and Non-beneficiaries had credibility and ranked I to the Input dealers. It is may be because in study area extension linkage between agricultural officers and farmer was not very good. Therefore it is needed to create awareness and good linkage between farmers and extension personals provision technical knowledge to farmers through training demonstration or other programmes to improving knowledge level of the farmers on recommended technologies.

\section{References}

Niranjan H.K., Sharma H.O. and Rathi D. 2016 Impact of national food security mission (NFSM) on annual usage and benefit derived from farm equipment in cultivation of wheat in Madhya Pradesh. International Journal of Agriculture 
Sciences, Volume 8, Issue 10, 2016, pp.-1116-1118.

Painkra V.K. 2014 Assessment of technological gap in production of black gram among tribal farmers of Jashpur district (Chhattisgarh). M.Sc. (Ag.) Thesis, IGKV, Raipur, Chhattisgarh.

Pandey A. 2015 Study on seed management pattern among the tribal farmers of Northern hills Agro-Climatic Zone of Chhattisgarh state. M.Sc. (Ag.) Thesis, IGKV, Raipur, Chhattisgarh.

Sekhar C.S.C. and Y. Bhatt 2012 Possibilities and constraints in pulses production in India and impact of national food security mission, Final report, Institute of economic growth university of Delhi enclave.

Singh J.M. and Grover D.K. (2015) Impact of national food security mission-pulses on legumes production performance in Punjab, India Agricultural Research Communication Centre legume research, 38 (5): 609-615

Singh V. 2017 A study on impact of National food security mission (NFSM) on productivity and income among the chickpea beneficiaries in Bemetara and Mungeli District of Chhattisgarh. M.Sc. (Ag) thesis, Department of Agricultural Extension college of Agriculture faculty of agriculture Indira Gandhi Krishi Vishwavidyalaya Raipur.

\section{How to cite this article:}

Akanksha Pandey and Awasthi, H.K. 2018. Utilization Pattern and Credibility upon Different Information Sources by the NFSM Beneficiaries and Non-Beneficiaries Farmers in Northern Hills Agro-Climatic Zone of Chhattisgarh. Int.J.Curr.Microbiol.App.Sci. 7(09): 3676-3686. doi: https://doi.org/10.20546/ijcmas.2018.709.457 